Coupling effect of Brownian motion and laminar shear flow on colloid coagulation: a Brownian dynamics simulation study

This article has been downloaded from IOPscience. Please scroll down to see the full text article.

2012 Chinese Phys. B 21054702

(http://iopscience.iop.org/1674-1056/21/5/054702)

View the table of contents for this issue, or go to the journal homepage for more

Download details:

IP Address: 159.226.231.80

The article was downloaded on 11/12/2012 at 08:51

Please note that terms and conditions apply. 


\title{
Coupling effect of Brownian motion and laminar shear flow on colloid coagulation: a Brownian dynamics simulation study*
}

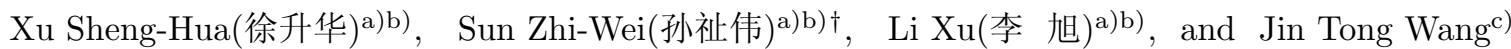 \\ a) Key Laboratory of Microgravity Institute of Mechanics, Chinese Academy of Sciences, Beijing 100190, China \\ b) National Microgravity Laboratory, Institute of Mechanics, Chinese Academy of Sciences, Beijing 100190, China \\ c) Department of Physics, Southern University and A\&M College, Baton Rouge, LA 70813, USA
}

(Received 12 October 2011; revised manuscript received 8 November 2011)

Simultaneous orthokinetic and perikinetic coagulations (SOPCs) are studied for small and large Peclet numbers $\left(P_{\mathrm{e}}\right)$ using Brownian dynamics simulation. The results demonstrate that the contributions of the Brownian motion and the shear flow to the overall coagulation rate are basically not additive. At the early stages of coagulation with small Peclet numbers, the ratio of overall coagulation rate to the rate of pure perikinetic coagulation is proportional to $P_{\mathrm{e}}^{1 / 2}$, while with high Peclet numbers, the ratio of overall coagulation rate to the rate of pure orthokinetic coagulation is proportional to $P_{\mathrm{e}}^{-1 / 2}$. Moreover, our results show that the aggregation rate generally changes with time for the SOPC, which is different from that for pure perikinetic and pure orthokinetic coagulations. By comparing the SOPC with pure perikinetic and pure orthokinetic coagulations, we show that the redistribution of particles due to Brownian motion can play a very important role in the SOPC. In addition, the effects of redistribution in the directions perpendicular and parallel to the shear flow direction are different. This perspective explains the behavior of coagulation due to the joint effects of the Brownian motion (perikinetic) and the fluid motion (orthokinetic).

Keywords: simultaneous orthokinetic and perikinetic coagulation, Brownian motion, shear flow, colloidal aggregation

PACS: 47.57.eb, 47.57.J-, 82.70.Dd, 82.20.Wt

DOI: $10.1088 / 1674-1056 / 21 / 5 / 054702$

\section{Introduction}

The coagulation kinetics and stability of colloidal suspensions has long been a major research field in colloid science. ${ }^{[1-10]}$ Coagulation depends on the transport of particles that induces particle collisions and the interparticle forces that determine the proportion of the collisions resulting in aggregation. The two basic transport mechanisms are Brownian diffusion and fluid motion. The coagulations caused by these two transport mechanisms are called perikinetic and orthokinetic coagulations, respectively.

The theoretical foundation for both perikinetic and orthokinetic coagulation was formulated by Smoluchowski. ${ }^{10]}$ In his classical treatment of orthokinetic coagulation, particle motion was considered to be caused by the laminar shear only. However, no pure orthokinetic coagulation can actually exist, because Brownian motion is always present, regardless of whether the shear flow exists or not. When the shear flow does exist, coagulation should be simultaneously orthokinetic and perikinetic.

Swift and Friedlander ${ }^{[11]}$ analysed the kinetics of simultaneous orthokinetic and perikinetic coagulation (SOPC) by assuming that the contributions from the two mechanisms were additive. They found some supportive experimental data of the coagulation of polystyrene particles for their additive assumption. The additive approximation was convenient to use, so it was adopted and discussed in some subsequent studies. ${ }^{[12-14]}$ However, the assumption was criticized by some researchers. ${ }^{[15-18]}$ Van de Ven and Mason ${ }^{[16]}$ theoretically studied the SOPC of small Peclet numbers. Their results showed that there is no theoretical foundation for the additive assumption. Feke and Schowalter ${ }^{[17]}$ pointed out that the additive assumption is inconsistent with the asymptotic corrections for solid spheres with very small and very large Peclet

* Project supported by the National Natural Science Foundation of China (Grant Nos. 10972217, 10932012 and 11032011) and the Knowledge Innovation Program of the Chinese Academy of Sciences (Grant No. KJCX2-YW-L08).

${ }^{\dagger}$ Corresponding author. E-mail: sunzw@imech.ac.cn

(C) 2012 Chinese Physical Society and IOP Publishing Ltd

http://iopscience.iop.org/cpb http://cpb.iphy.ac.cn 
numbers. In addition, there could be a strong coupling between the flow strength and the effect of Brownian motion. The collision rates of spherical drops or particles were also studied by using the Fokker-Plank equation for a broad range of Peclet numbers. ${ }^{[18]}$ It was concluded that the additive approximation is usually questionable and a modified approximation provides better results.

In those theoretical studies, only the early stages of coagulation were considered, and the obtained results were conflicting, suggesting that the SOPC is rather complicated, even for coagulations at the early stages. The SOPC could be even more complicated for coagulations beyond the early stage. Therefore, it is necessary to investigate the coupling effect of Brownian motion and shear flow, which may provide better understandings of the applicability of different theories and the mechanism for the possible change of the coagulation rate during the SOPC. A useful strategy for this purpose is to study the behavior of SOPC and compare it with that of pure perikinetic coagulation (PPC) and pure orthokinetic coagulation (POC).

Computer simulation provides a simple method to study the complicated SOPC process. In simulations, the effect of the Brownian motion can be easily turned on or off, which is impossible in an experiment. So a computer simulation makes it possible to directly compare SOPC with POC and PPC. Moreover, effects other than Brownian motion and shear flow, such as hydrodynamic interaction, can be neglected in the simulation, so we can concentrate on the influences of these two mechanisms on the aggregation behavior without any interference.

Brownian dynamics (BD) simulation has been proved to be a useful approach to studying the aggregation of colloidal particles. ${ }^{[19-21]}$ In this study, we will investigate the SOPC with different parameters using the BD simulation. Since our goal is to study the influence of the transport mechanism on the coagulation process, we do not include particle interactions, and the particles are assumed to aggregate upon collision. Similar to what was done in the previous $\mathrm{BD}$ studies on colloidal coagulation, ${ }^{[19-21]}$ the hydrodynamic interactions are also neglected. The results are compared with those of POC and PPC to study how Brownian motion and the laminar shear affect the SOPC process. The additive approximation and the other theoretical predictions are also compared with the simulated results for different Peclet numbers, which gives the conditions under which the theories are applicable and are helpful for the future analysis of coagulation through Brownian motion and shear flow.

\section{Simulation}

BD simulation is utilized in this study. In order to concentrate on the influence of transport mechanisms on colloidal aggregation and to make comparisons with the theories in which the hydrodynamic interactions are ignored, we neglect the hydrodynamic interactions in the $\mathrm{BD}$ simulation, which is similar to what has been done in previous BD studies of colloidal aggregation. ${ }^{[19-21]}$ Such a treatment also saves simulation time, as BD simulation considering the hydrodynamic interactions ${ }^{[22-24]}$ can be very time consuming when the particle number in the system is large.

The Langevin equation for a system of $N$ nonhydrodynamically interacting Brownian particles is

$$
m_{i} \dot{v}_{i}(t)=-\zeta v_{i}(t)+F_{i}(t)+p_{i}^{\mathrm{G}}(t),
$$

where $i$ represents the $i$-th component $(1 \leq i \leq 3 N)$; $v$ and $m$ represent the velocity and the mass of the particle, respectively; $F$ is the external force acting on the particle; $-\zeta v_{i}(t)$ is the friction force; and $p_{i}^{\mathrm{G}}(t)$ is a random term representing the effect of Brownian motion. The algorithm for the $\mathrm{BD}$ simulation based on the Langevin equation can then be written as ${ }^{[1,22,25]}$

$$
r_{i}(t+\Delta t)=r_{i}(t)+\frac{D_{i} F_{i}}{k T} \Delta t+\Delta r_{i}^{\mathrm{G}},
$$

where $k$ is the Boltzmann constant, $T$ is the temperature, $D_{i}$ is the diffusion coefficient, $\Delta t$ is the time step, and $\Delta r_{i}^{\mathrm{G}}$ has a Gaussian distribution with zero mean and variance $2 D_{i} \Delta t$. Here, $D_{i}$ equals $k T /\left(6 \pi \eta a_{i}\right)$ with $\eta$ being the viscosity coefficient and $a_{i}$ the radius of particle $i$.

In this study, we focus only on the coagulation process caused by particle transport. Therefore, the external force $F_{i}$ is zero. If shear flow exists in the system, the particle will also move with the fluid motion at a velocity depending on the position of the particle. Then the algorithm used in this study can be written as

$$
r_{i}(t+\Delta t)=r_{i}(t)+v_{i}^{\mathrm{S}}(t) \Delta t+\Delta r_{i}^{\mathrm{G}},
$$

where $v_{i}^{\mathrm{S}}$ is the velocity of the particle depending on the shear flow.

The shear flow used in the simulation is laminar Couette flow, which is similar to that in Smoluchoski's theory. The flow velocity is a function of $y$, and the 
flow direction is along the $x$ axis in the simulation. The flow field can be expressed as

$$
v_{x}(y)=G|y|
$$

where $-L / 2 \leq y \leq L / 2$ with $L$ being the size of the simulation box, and $G$ is the shear rate.

At the beginning of the simulation, we randomly place $N_{0}=20000$ monodispersed particles without superposition in the simulation box. The particle diameter $d$ is $1.0 \mu \mathrm{m}$, and the size of the simulation box $L$ is $1.25 \mathrm{~mm}$, so the number concentration of the system is $1.024 \times 10^{7} \mathrm{~cm}^{-3}$, and the volume fraction $\varphi$ is about $5.36 \times 10^{-6}$. The temperature in the simulation is $T=300 \mathrm{~K}$. The other two important parameters, viscosity coefficient $\eta$ and shear rate $G$, will be changed in the study to adjust the particle transport caused by Brownian motion and fluid motion. Using these parameters, the particle velocity caused by the shear flow and the diffusion coefficient can be calculated as described above. Then, the motion of the particles caused by Brownian motion and the shear flow can be calculated iteratively by using Eq. (3). If $v_{i}^{\mathrm{S}}(t)\left(\Delta r_{i}^{\mathrm{G}}\right)$ is taken to be zero in Eq. (3), we can also simulate PPC (POC) by using BD simulation.

In the simulation, when the distance between two particles (or aggregates) is smaller than the sum of their radii, the two particles (or aggregates) are considered to collide and aggregate. Since the volume fraction is very low, a multi-body collision will not occur in the simulation. Therefore, after each aggregation the particle number decreases by one and the two colliding particles (or aggregates) form a larger aggregate. For simplicity, we assume that the newly formed aggregate is spherical with a volume equal to the total volume of the two colliding particles or aggregates. By this means, the particle numbers at different aggregation times can be obtained for different parameters. And the results are used to analyze the coagulation processes of POC, PPC, and SOPC, respectively, as will be shown in the following Sections.

\section{Results and discussion}

\subsection{POC and PPC}

When the hydrodynamic interactions are ignored, according to Smoluchowski's theory, the rate constant for the collisions of two same-sized particles for perikinetic coagulation is ${ }^{[1,10]}$

$$
k_{\text {peri }}=\frac{8 k T}{3 \eta} .
$$

For the collisions of different-size particles, the rate constant will be a little different from Eq. (5). However, all the rate constants are usually assumed to be equal in theory, so the change of the concentration of particle can be written as ${ }^{[1]}$

$$
\frac{\mathrm{d} N}{\mathrm{~d} t}=-\frac{k_{\text {peri }} N^{2}}{2} .
$$

With the initial condition of $N=N_{0}$ at coagulation time $t=0$, the particle number concentration for perikinetic coagulation can be expressed as

$$
N=\frac{N_{0}}{1+k_{\text {peri }} N_{0} t / 2} .
$$

Figure 1 shows the simulation results of the residual particle numbers in the simulation box for PPC, the theoretical results from Smoluchowski are also shown for comparison. The values of viscosity coefficient $\eta$ used are $1 \times 10^{-3} \mathrm{~kg} \cdot \mathrm{m}^{-1} \cdot \mathrm{s}^{-1}$ and $2 \times 10^{-3} \mathrm{~kg} \cdot \mathrm{m}^{-1} \cdot \mathrm{s}^{-1}$, respectively. The time step in the simulation is $5 \times 10^{-3} \mathrm{~s}$. The figure shows that the simulation results are close to the theoretical ones. The results also show that our method is suitable for simulating the coagulation process of colloidal particles.

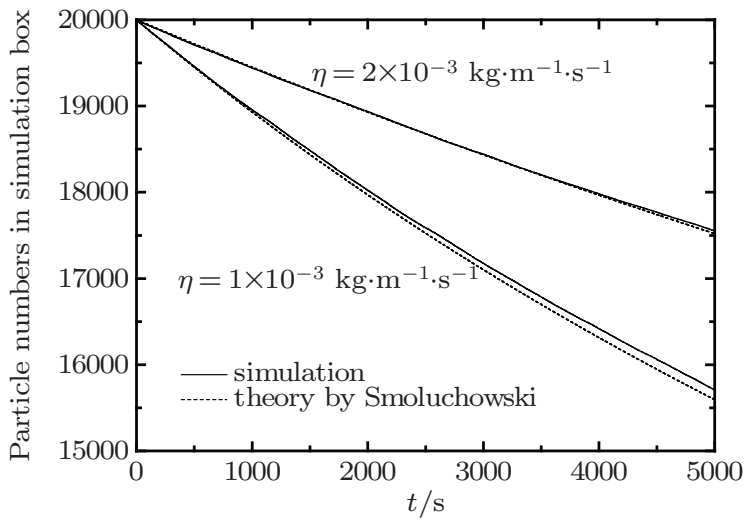

Fig. 1. Simulation results of PPC compared to Smoluchowski's theoretical results (Eq. (7)).

For POC caused by the laminar shear, according to Smoluchowski's theory, the rate constant of the collisions between two particles of radius $a$ is ${ }^{[1,10]}$

$$
k_{\text {ortho }}=\frac{32 G a^{3}}{3} \text {. }
$$

By analogy with Eq. (6), the differential equation for $\mathrm{POC}$ is

$$
\frac{\mathrm{d} N}{\mathrm{~d} t}=-\frac{16}{3} N^{2} G a^{3}=-\frac{4 N G}{\pi} \varphi,
$$


where the volume is $\varphi=4 \pi N a^{3} / 3$. The particle number concentration during the orthokinetic coagulation can be deduced from Eq. (9) as ${ }^{[1]}$

$$
N=N_{0} \exp \left(-\frac{4 G \varphi t}{\pi}\right) .
$$

The calculation results of Eq. (10) are compared with the simulation results for the POC in Fig. 2. For the shear rates of $0.1 \mathrm{~s}^{-1}$ and $0.4 \mathrm{~s}^{-1}$, the time step in the simulation is $5 \times 10^{-3} \mathrm{~s}$. For larger shear rates, we reduce the time step to make the simulation reasonable. The time step is $5 \times 10^{-4} \mathrm{~s}$ for the shear rates of $60 \mathrm{~s}^{-1}, 80 \mathrm{~s}^{-1}$, and $100 \mathrm{~s}^{-1}$. Since the time step is reduced and the total steps in the simulation are kept the same, the coagulation time is also reduced for larger shear rates, as shown in Fig. 2.
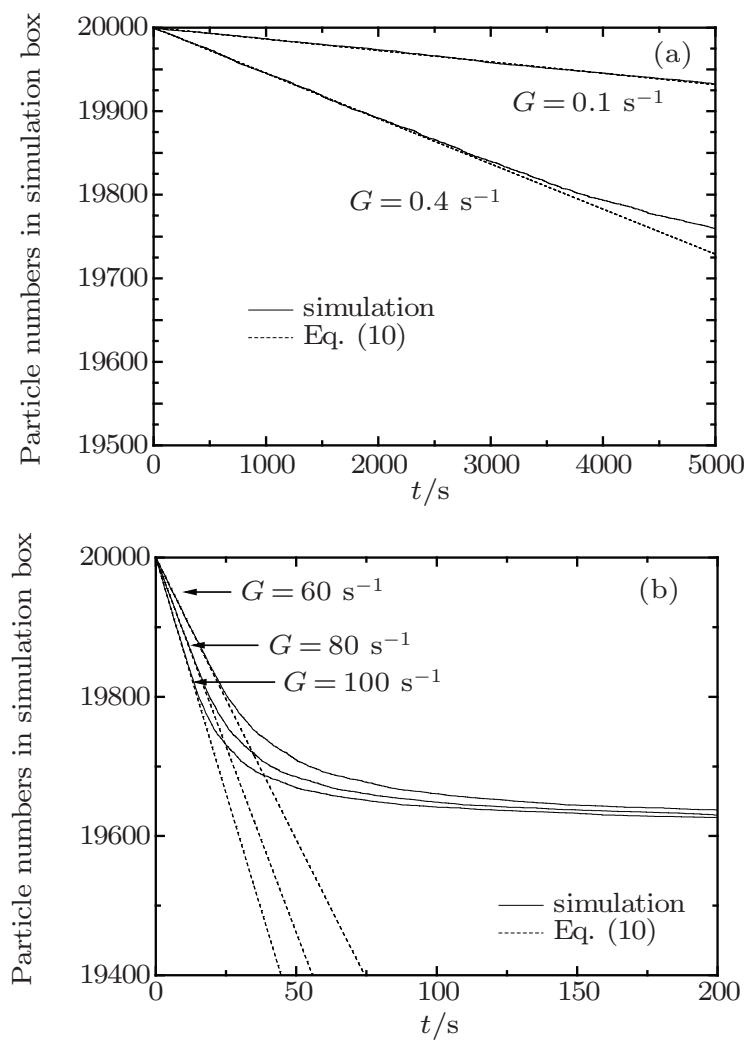

Fig. 2. Simulation results of POC compared to Smoluchowski's theoretical results (Eq. (10)) for (a) small and (b) large shear rates.

Figure 2 shows that there are significant deviations between the simulation results and the theoretical ones when the residual particle numbers drop down to certain values (about 19800 in Fig. 2). Such deviations are caused by the periodic boundary conditions used in the simulation. For POC, the particles move only in one dimension, that is, along the direction of the shear flow, so two particles $i$ and $j$ can aggregate only when $\sqrt{\left(y_{i}-y_{j}\right)^{2}+\left(z_{i}-z_{j}\right)^{2}}<\left(a_{i}+a_{j}\right)$.
The $y$ and $z$ coordinates of the particles cannot be changed by the shear flow along the $x$ axis. Due to the periodic boundary condition, the particle distribution is only uniform in a box of size $L$, but not in an unbounded domain. Therefore, after some coagulation time when most of the particle pairs satisfying $\sqrt{\left(y_{i}-y_{j}\right)^{2}+\left(z_{i}-z_{j}\right)^{2}}<\left(a_{i}+a_{j}\right)$ have already aggregated, the remaining particles will have very low aggregation probabilities. Comparatively, Brownian motion can make the particles distribute randomly and uniformly in three dimensions, so the periodic boundary condition has little influence on PPC, as shown in Fig. 1.

According to the periodic boundary condition, the long-time limit number of particles that can possibly aggregate in the POC can be easily estimated based on the probability of collision between particles, the value is given by $N_{0}\left(N_{0}-1\right) \pi d^{2} / L^{2}$. For the system used in this study, the value is approximately 800 , so the final number of particles for the POC will be about $20000-800+800 / 2=19600$, which is consistent with our simulation result shown in Fig. 2. To completely avoid the influence of the boundary condition on the POC, all particles in the system should have probabilities to aggregate, so value $N_{0}\left(N_{0}-1\right) \pi d^{2} / L^{2}$ needs to be larger than $N_{0}$. Therefore, $\left(N_{0}-1\right) \pi d^{2} / L^{2}$, which is approximately $6 \varphi L / d$, should be larger than 1 . If the volume fraction $\varphi$ and the particle diameter $d$ are kept unchanged, the simulation box needs to be increased by 25 times and the particle number should be about 0.3 billion to ensure $6 \varphi L / d \geq 1$. Such a huge number of particles are apparently far beyond the capability of our current computing facilities. Therefore, it would be very difficult to study the long-time behavior of SOPC with a large Peclet number without the influence of the periodic boundary condition. However, for each shear rate, there is still a time interval at the beginning of the coagulation during which the boundary condition has an ignorable influence. In that time interval, the simulation results are very close to the theoretical ones, as shown in Fig. 2. In order to avoid the influence of the boundary conditions in the following discussion about the SOPC, the time intervals determined from the results shown in Fig. 2 will be used.

The simulated results in Figs. 1 and 2 and the relevant discussion in Section 2 show that the $y$ and $z$ coordinates of the particles are never changed by the shear flow along the $x$ axis, while Brownian motion can make the coordinates uniformly distribute in three 
dimensions. For SOPC, the redistribution of particles by Brownian motion may greatly influence the coagulation caused by the shear rate. Because of the redistribution of coordinates along the $y$ and $z$ axes, the particles not satisfying $\sqrt{\left(y_{i}-y_{j}\right)^{2}+\left(z_{i}-z_{j}\right)^{2}}<$ $\left(a_{i}+a_{j}\right)$ can aggregate by the shear flow. Such an effect tends to cause more particles to aggregate under the shear flow. Also, particle pairs will become gradually closer in the $x$ direction for POC until they eventually collide and aggregate. Then, the redistribution along the $x$ axis tends to cause these pairs to disperse, which will reduce the aggregation rate by shear flow. These two effects will be discussed in the following Sections.

\subsection{SOPC with small Peclet numbers}

A simple theory to deal with SOPC is based on the additive assumption, ${ }^{[11]}$ in which the collision rates for Brownian motion and laminar shear flow are assumed to be additive. Therefore, with Eqs. (5), (6), and (9), the change of the total particle concentration with time for SOPC is given by

$$
\begin{aligned}
\frac{\mathrm{d} N}{\mathrm{~d} t} & =-\frac{16}{3} N^{2} G a^{3}-\frac{4 k T}{3 \eta} N^{2} \\
& =-\frac{4 N G}{\pi} \varphi-\frac{4 k T}{3 \eta} N^{2} .
\end{aligned}
$$

From the above equation, we can obtain ${ }^{[11]}$

$$
\ln \left[\left(\frac{N+P}{N}\right)\left(\frac{N_{0}}{N_{0}+P}\right)\right]=\frac{4 G \varphi}{\pi} t,
$$

where $P=3 G \varphi \eta / \pi k T$. Then the particle number concentration $N$ at coagulation time $t$ can be expressed as

$$
N=\frac{P}{\left(\frac{N_{0}+P}{N_{0}}\right) \exp \left(\frac{4 G \varphi}{\pi} t\right)-1}
$$

Equation (11) indicates that the rate constant for the SOPC collisions is

$$
k_{\mathrm{SOPC}}=\frac{32 G a^{3}}{3}+\frac{8 k T}{3 \eta}=16 \pi D a\left(1+\frac{2}{3 \pi} P_{\mathrm{e}}\right),
$$

where $P_{\mathrm{e}}$ is the Peclet number and is given by

$$
P_{\mathrm{e}}=\frac{G a^{2}}{D}=\frac{6 \pi \eta G a^{3}}{k T} .
$$

However, the correctness of the additive assumption is questionable. For small Peclet numbers,
Ref. [16] shows that the increment of the coagulation rate relative to the perikinetic coagulation due to the shear is on the order of $P_{\mathrm{e}}^{1 / 2}$ instead of $P_{\mathrm{e}}$ shown in Eq. (14). When there is no interparticle force, as in this study, the coagulation rate should be expressed as $^{[16]}$

$$
k_{\mathrm{SOPC}}=16 \pi D a\left(1+0.5136 P_{\mathrm{e}}^{1 / 2}\right) .
$$

Thus, Eq. (11) should be written as

$$
\frac{\mathrm{d} N}{\mathrm{~d} t}=-8 \pi D a\left(1+0.5136 P_{\mathrm{e}}^{1 / 2}\right) N^{2} .
$$

Then we can obtain

$$
N=\frac{N_{0}}{1+8 \pi D a\left(1+0.5136 P_{\mathrm{e}}^{1 / 2}\right) N_{0} t}
$$

Equations (13) and (18) give the particle number concentrations for SOPC based on the additive assumption and the theory in Ref. [16], respectively. Both theoretical results are compared with our simulation results for some small Peclet numbers in Fig. 3. For the simulation shown in Fig. 3, the time step used is $5 \times 10^{-3}$ s. Since Eqs. (13) and (18) are deduced from the rate constants of collisions at the initial stages of coagulation, the comparisons for the early stages are also shown in Fig. 3.

Figures 3(a) and 3(c) show the results of $5000 \mathrm{~s}$ and 3000 s coagulations for $G=0.1$ and 0.4 , respectively. As discussed in Section 2, at these time intervals, the boundary condition has an ignorable influence. Figures 3(b) and 3(d) correspondingly show the results for the early stages (first $100 \mathrm{~s}$ ). Since in this section we study SOPC with low values of $P_{\mathrm{e}}$, the values of $P_{\mathrm{e}}$ in Fig. 3 are all smaller than 1 . The viscosity coefficients are $1 \times 10^{-3} \mathrm{~kg} \cdot \mathrm{m}^{-1} \cdot \mathrm{s}^{-1}$ and $2 \times 10^{-3} \mathrm{~kg} \cdot \mathrm{m}^{-1} \cdot \mathrm{s}^{-1}$ in Fig. 3. The corresponding $P_{\mathrm{e}}$ in these figures are $0.0569,0.114,0.228$, and 0.455 respectively. Figure 3 clearly shows that the theory in Ref. [16] is very precise for the early stages of SOPC with small $P_{\mathrm{e}}$. The additive assumption based theory underestimates the coagulation rate, so it is not accurate for SOPC, even at the early stages. This result is consistent with the conclusions obtained in the above Section. Brownian motion can redistribute the particles, so it can increase the aggregation. Such an additional contribution makes the aggregation rate larger than the summation of PPC and POC (additive assumption) at the initial stages of coagulation. 

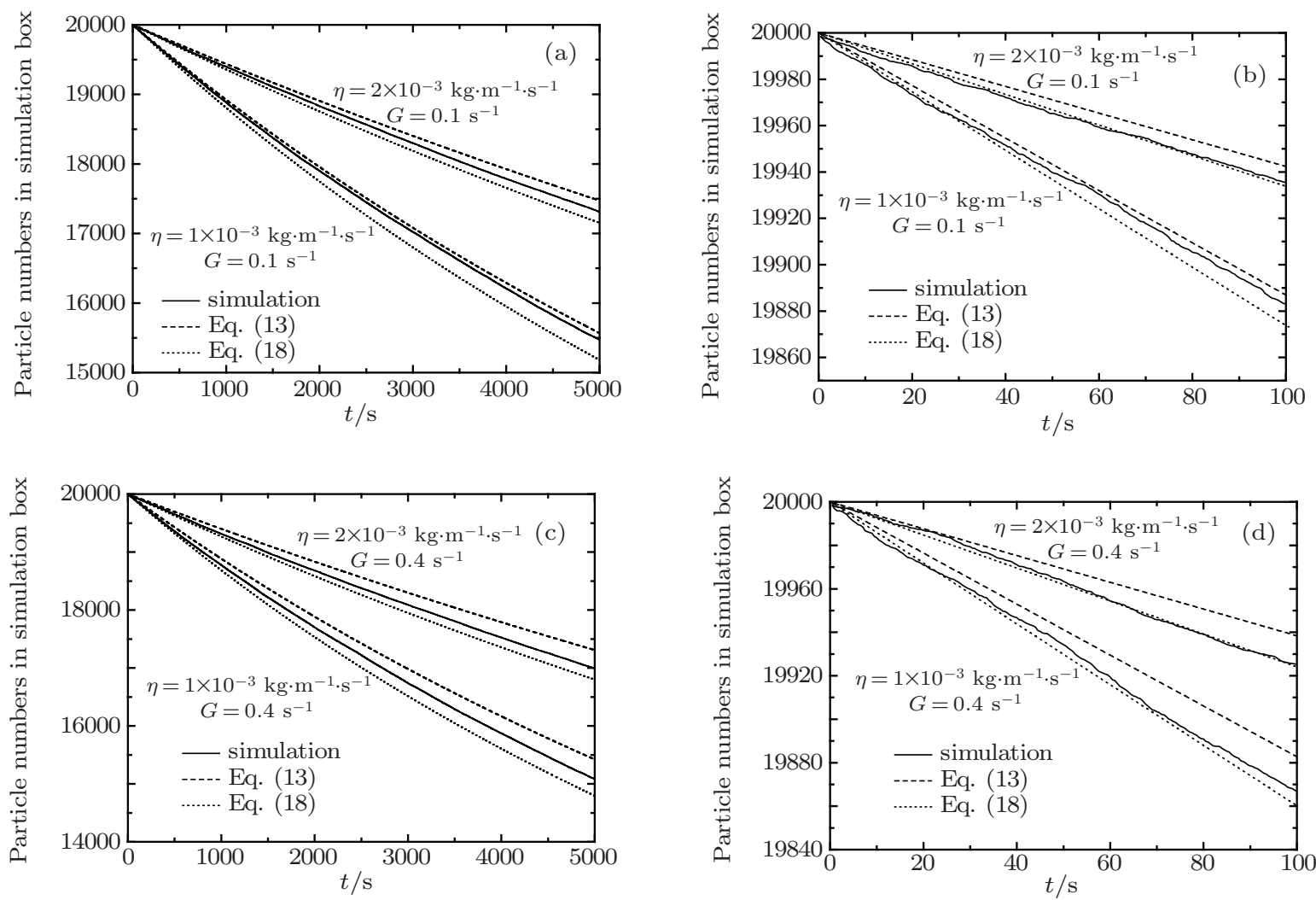

Fig. 3. Simulation results of SOPC with small Peclet numbers compared to results obtained from Eqs. (13) (additive assumption) and (18) (theory in Ref. [16]). Panels (a) and (c) show the results for different parameters during long time of coagulation. Panels (b) and (d) exhibit the results for the first $100 \mathrm{~s}$ of coagulation.

Although Eq. (18) can predict the coagulation rates at the early stages of coagulation, the predicted particle numbers are smaller than the simulated results for coagulation beyond the early stages, as shown in Figs. 3(a) and 3(c). Such a difference indicates that the aggregation rates decrease with time. This is caused by the redistribution along the $x$ axis that tends to reduce the aggregation rate as described in the above section. As time passes, the effect of redistribution at the $x$ axis becomes obvious, causing the aggregation to be slower at the later stages than that at the early stages.

In summary, Fig. 3 shows that the theory in Ref. [16] can successfully describe the coagulation feature for the early stages of SOPC with small $P_{\mathrm{e}}$. However, the aggregation rate decreases with the increasing time, so the theory cannot fit the aggregation beyond the early stages very well. The redistribution of particle positions by Brownian motion plays an essential role in SOPC with small $P_{\mathrm{e}}$.

\subsection{SOPC with large Peclet numbers}

The simulation results of SOPC with large Peclet numbers are compared with the theoretical ones un- der the additive assumption in Fig. 4. In Fig. 4(a), the viscosity coefficient is $\eta=2 \times 10^{-3} \mathrm{~kg} \cdot \mathrm{m}^{-1} \cdot \mathrm{s}^{-1}$, and the Peclet numbers are 68.3, 91.1, and 114 respectively. While in Fig. 4(b), $\eta=1 \times 10^{-3} \mathrm{~kg} \cdot \mathrm{m}^{-1} \cdot \mathrm{s}^{-1}$, and the Peclet numbers are $34.1,45.5$, and 56.9 respectively. For these Peclet numbers, the shear rates are so high that the boundary condition has an ignorable influence only for the very short time indicated in Fig. 2. Therefore, only the initial stages are discussed in this section.

Figure 4 shows that the additive assumption based theory overestimates the number of aggregated particles compared with the simulated results at the early stages. For high Peclet numbers, the motion caused by the shear flow along the $x$ axis dominates. The redistribution of particles along the $x$ axis by Brownian motion is more important than that along the $y$ and $z$ axes to affect the aggregation caused by the shear. This effect reduces the total aggregation rate of SOPC, making it lower than that with the additive assumption.

According to Ref. [18], the effects of Brownian motion and the shear rate should not be additive, and the ratio between the rate constants of two spherical drops or particles for SOPC and POC is proportional 
to $P_{\mathrm{e}}^{-1 / 2}$ for high Peclet numbers. According to this theory, we can obtain

$$
k_{\mathrm{SOPC}} / k_{\text {ortho }}=C_{1}+C_{2} P_{\mathrm{e}}^{-1 / 2},
$$

where $k_{\mathrm{SOPC}}$ and $k_{\text {ortho }}$ are the coagulation rates for SOPC and POC, respectively.
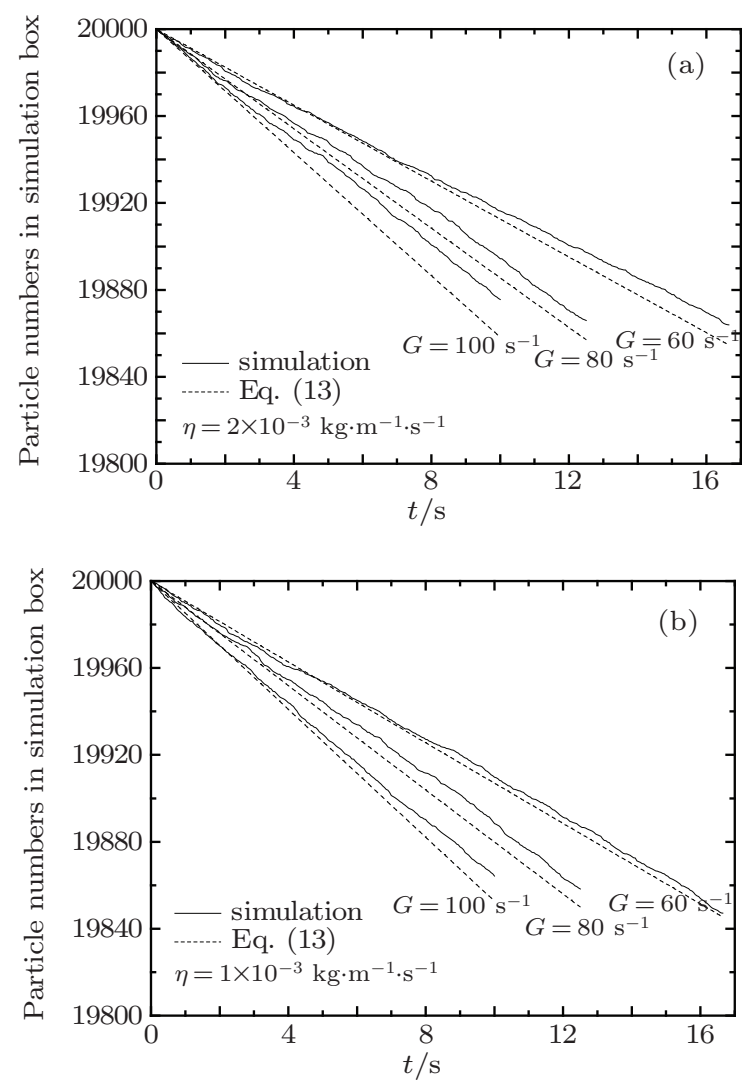

Fig. 4. Results of Eq. (13) (additive assumption) compared to simulation results for SOPC with high Peclet numbers. Panels (a) and (b) show results with different viscosity coefficients.

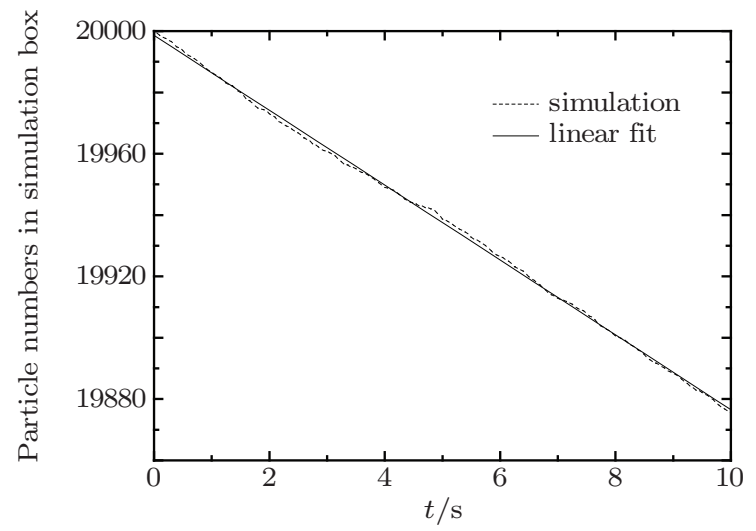

Fig. 5. Typical linear fitting for the simulated results of the initial stages of SOPC with high Peclet numbers, viscosity coefficient $\eta=2 \times 10^{-3} \mathrm{~kg} \cdot \mathrm{m}^{-1} \cdot \mathrm{s}^{-1}$, and shear rate $G=100 \mathrm{~s}^{-1}$.

Based on our simulation results, the values of $k_{\text {SOPC }}$ at different parameters can be determined by linearly fitting the data at the early coagulation stages. The typical fitting results are shown in Fig. 5 for $G=100 \mathrm{~s}^{-1}$ and $\eta=2 \times 10^{-3} \mathrm{~kg} \cdot \mathrm{m}^{-1} \cdot \mathrm{s}^{-1}$. The value of $k_{\text {ortho }}$ can be determined by using Eq. (8). After the determination of $k_{\mathrm{SOPC}}$ and $k_{\text {ortho }}$, a plot of $k_{\mathrm{SOPC}} / k_{\text {ortho }}$ versus $P_{\mathrm{e}}^{-1 / 2}$ can be obtained. For the simulation results shown in Fig. 4 , the $k_{\mathrm{SOPC}} / k_{\text {ortho }}$ versus $P_{\mathrm{e}}^{-1 / 2}$ is shown in Fig. 6. The result supports Eq. (19).

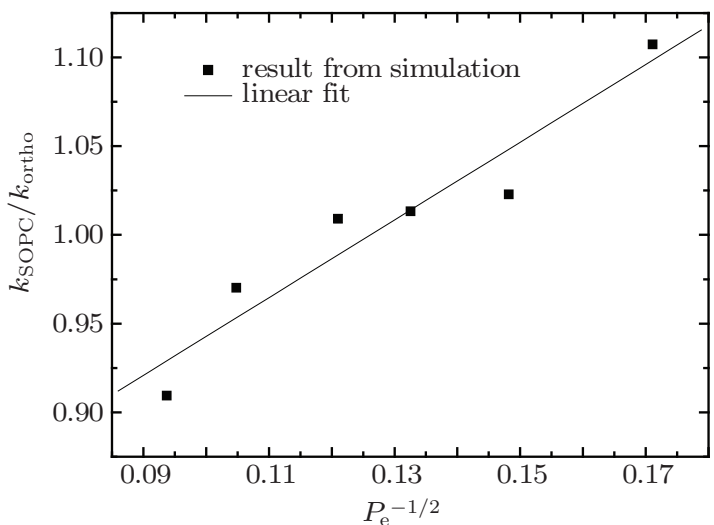

Fig. 6. The $k_{\mathrm{SOPC}} / k_{\text {ortho }}$ versus $P_{\mathrm{e}}^{-1 / 2}$.
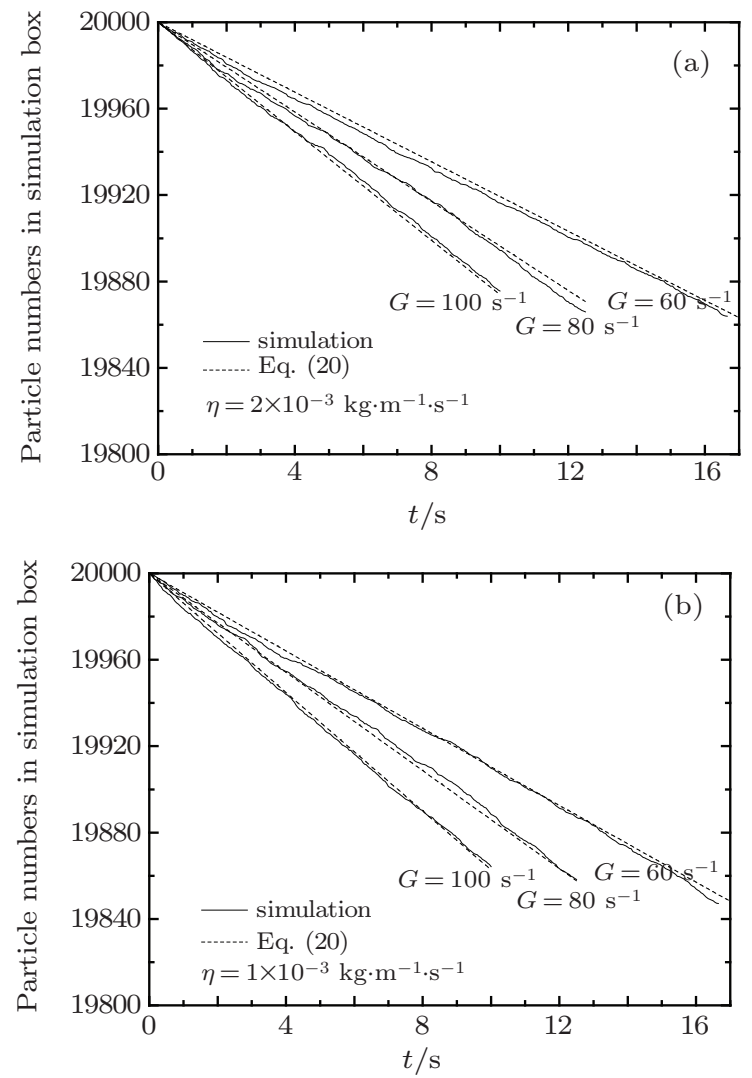

Fig. 7. Results of Eq. (20) (theory in Ref. [18]) compared to the simulation results of SOPC with large Peclet numbers. Panels (a) and (b) show results with different viscosity coefficients.

As shown in Fig. 6, the values of $k_{\mathrm{SOPC}}$ for 
different $P_{\mathrm{e}}$ can be evaluated theoretically by using Eq. (19). Similar to the deduction of Eq. (18), the number concentration can then be evaluated from the theoretically evaluated $k_{\mathrm{SOPC}}$ as

$$
N=\frac{N_{0}}{1+k_{\mathrm{SOPC}} N_{0} t / 2} .
$$

The results of Eq. (20) are compared with the simulated results of SOPC in Fig. 7. In Fig. 7(a), the viscosity coefficient is $\eta=2 \times 10^{-3} \mathrm{~kg} \cdot \mathrm{m}^{-1} \cdot \mathrm{s}^{-1}$, while in Fig. $7(\mathrm{~b}), \eta=1 \times 10^{-3} \mathrm{~kg} \cdot \mathrm{m}^{-1} \cdot \mathrm{s}^{-1}$. Figure 7 shows that the particle numbers evaluated from Eq. (20) are close to the simulated results, showing that Eq. (20) is better than Eq. (13) for SOPC with large Peclet numbers.

\section{Conclusion}

By using Brownian dynamics simulation, we study SOPCs with small and large Peclet numbers, and the results are compared with different theoretical predictions. The applicabilities of different theories are tested directly, and the effects of Brownian motion and the laminar shear on SOPC are studied. Moreover, the results are explained by comparison with those of PPC and POC, which shows that the redistribution of particles can play an essential role in SOPC.

The simulation results provide direct evidences that the additive assumption is inappropriate even at the very initial stages of SOPC. For small Peclet numbers, the additive assumption based theory underestimates the aggregation rate, while Eqs. (16)-(18) are preferable. For large Peclet numbers, the additive assumption based theory overestimates the aggregation rate, and Eqs. (19) and (20) are preferable. Moreover, for small Peclet numbers, the simulated results in the later stages also show that the coagulation rate decreases during SOPC. All these results can be explained by the different effects of the redistribution (by Brownian motion) in the $y$ and $z$ axis directions (perpendicular to the flow direction) and in the $x$ axis direction (parallel to the flow direction).

Due to the influence of the periodic boundary condition, for SOPC with large Peclet numbers, only the initial stages in which the boundary condition has an ignorable influence can be studied. To essentially avoid the influence of the periodic boundary condition, the required particle number and the size of the simulation box are far beyond the capability of our current computing facilities. Future studies concerning the later stages of SOPC with large Peclet numbers will be beneficial for deepening our understanding of SOPC.

\section{References}

[1] Elimelech M, Gregory J, Jia X and Williams R A 1995 Particle Deposition and Aggregation (Oxford: Butterworth-Heinemann)

[2] Allain C, Cloitre M and Wafra M 1995 Phys. Rev. Lett. 741478

[3] Molina-Bolívar J A, Galisteo-González F and HidalgoÁlvarez R 1999 J. Chem. Phys. 1105412

[4] Stanislav D, Chao Z, Rajesh N D and Qun Y 2005 Adv. Colloid Interface Sci. 114119

[5] Vincent B 1992 Adv. Colloid Interface Sci. 42279

[6] Sun Z W, Xu S H, Liu J, Li Y M, Lou L R and Xie J C 2005 J. Chem. Phys. 122184904

[7] Sun Z W, Liu J and Xu S H 2006 Langmuir 224946

[8] Liu J, Xu S H and Sun Z W 2007 Langmuir 2311451

[9] Xu S H and Sun Z W 2010 Langmuir 266908

[10] Smoluchowski M 1917 Z. Phys. Chem. 92129

[11] Swift D L and Friedlander S K 1964 J. Colloid Sci. 19 621

[12] Laurenzi I J and Diamond S L 2002 Ind. Eng. Chem. Res. 41413

[13] Han M Y and Lawler D F 1992 J. Am. Water Works Assoc. $\mathbf{8 4} 79$

[14] Chang H N and Robertson C R 1976 Ann. Biomed. Eng. 4151

[15] Melis S, Verduyn M, Storti G, Morbidelli M and Baldyga J 1999 AIChE J. 451383

[16] van de Ven T G M and Mason S G 1977 Colloid Polymer Sci. 255794

[17] Feke D L and Schowalter W R 1983 J. Fluid Mech. 133 17

[18] Zinchenko A Z and Davis R H 1995 Phys. Fluids 72310

[19] Puertas A M, Maroto J A, Fernandez-Barbero A and de las Nieves F J 1999 Phys. Rev. E 591943

[20] Puertas A M, Fernandez-Barbero A and de las Nieves F J 1999 Comput. Phys. Commun. 121-122 353

[21] Romero-cano M S, Puertas A M and de las Nieves F J 2000 J. Chem. Phys. 1128654

[22] Ermak D L and McCammon J A 1978 J. Chem. Phys. 69 1352

[23] Banchio A J and Brady J F 2003 J. Chem. Phys. 118 10323

[24] Grayer T and Winter U 2009 J. Chem. Phys. 130114905

[25] Xu S H and Sun Z W 2007 J. Chem. Phys. 126144903 